2. Real Estate, Partners HealthCare, Somerville/MA/United States of America

3. Risk Management And Insurance, Partners HealthCare, Somerville/MA/United States of America

4. Emergency Preparedness, Partners HealthCare System, Boston/ MA/United States of America

Study/Objective: We designed a climate risk-assessment project that demonstrates value to leadership and expert stakeholders, and ultimately creates understanding of climate threats facing our health system, in order to implement effective interventions.

Background: Climate change is influencing weather intensity and patterns creating new, increased threats for health care facilities. Historical data are no longer sufficient in determining. risk, as evidenced by the 2016 Louisiana floods where one-third of flooding occurred outside of the 100-year flood zones. Health care organizations must consider the surrounding built environment and community networks, which could influence the impact of an extreme-weather event upon their operations. Conducting the detailed, forward-looking analysis required to make informed decisions requires broad leadership and subject matter, expert collaboration internal and external to the organization.

Methods: A multidisciplinary project team was formed comprised of senior leaders in real-estate, emergency preparedness, risk management, insurance, and external climate experts. Together, these representatives could address structural, operational and fiscal challenges and opportunities related to climate threats based in science. Three data collection tools were chosen: (1) detailed, multi-scenario climate modeling; (2) completion of a climate-resilient health care facilities checklist; and (3) stakeholder meetings with insurers, public utilities, and public transportation agencies to understand external vulnerabilities and opportunities. Finally, analysis was conducted with near and long-term horizons, allowing twopoints of intervention: operational changes in the near-term, and facility construction changes addressing long-term threats. Results: Phase I of the project was completed for 30 sites across the health system. Results were shared with key leaders at the enterprise and institution level. Key findings include a system-wide threat from extreme heat events and vulnerabilities to critical infrastructure which may place an indirect burden on our facilities.

Conclusion: Building climate resiliency requires a multidisciplinary approach. Assessed at multiple time horizons, facilities upgrades, operational enhancements, and improved coordination with interdependent agencies and institutions can occur.

Prehosp Disaster Med 2017;32(Suppl. 1):s66-s67

doi:10.1017/S1049023X17001807

\section{A Proposed Disaster Casualty Classification Framework} Guo H. Tao

Department Of Health Service, Logistics University of PAP, Tianjin/ China

Study/Objective: To design a disaster-casualty classification framework.
Background: "Casualty" is a key term in the discipline of disaster medicine. Searching and rescuing disaster casualties is the main work of a health care task force in a disaster zone. However, the term is often erroneously used for the seriously injured and dead. Until the term "casualty" had been clearly defined and classified, we couldn't get a full picture of casualty flow in disasters. There is a difference in managing patients with treatable traumas and diseases versus those who struggle resulting in death.

Methods: Multiple web searching tools (Pubmed, Wikipedia, Yahoo search, etc.) were used for relevant articles, abstracts, and grey literatures covering the period January 2000-December 2015. A qualitative survey questionnaire was designed based on search results. An informal, multi-disciplinary, expert working group was established, including 18 individuals representing the discipline of emergency management, public health, clinical medicine, and military medicine. The experts were invited to write comments on the questionnaire separately. The comments of the experts were synthesized into a comprehensive report. In July of 2016, an expert meeting was held on our campus to discuss the report and reach a consensus about the disaster-casualty classification framework.

Results: Eleven documents were considered highly relevant. The experts believed that before giving a definition to "casualty," "disaster scene" and "health care facility in the disaster zone" should be defined. We then define "casualty" as "anyone incurring a trauma or illness, or dying as a direct result of disaster." Disaster casualty must include the deceased and can be classified into two parts: casualties with trauma and casualties with illness. Each part has three sub-groups: death on the scene, casualty coming to a health care facility for treatment, and casualty who needs medical treatment but didn't come to any health care facility. For casualty coming to a health care facility for treatment, it can be classified into three portions: death in the health care facility, the inpatient, and the outpatient. Each associated term must be defined carefully and explicitly. Disaster casualty has its unique classification method; each part and sub-group need different public health and medical interventions and treatments.

Conclusion: This is a tentative study to draw a picture of disaster casualty. Disaster exerts tremendous influence on disaster casualty and the process of casualty production is complex and complicated. Our disaster-casualty classification framework is proposed to be tested and improved.

Prehosp Disaster Med 2017;32(Suppl. 1):s67

doi:10.1017/S1049023X17001819

The Challenges on Implementation of Pre-Disaster Efforts of Health Crisis Center (PPKK), Indonesia Ministry of Health $(\mathrm{MoH})$ in 2014

Sukma M. Panggabean ${ }^{1}$, Yuli Amran ${ }^{2}$, Mochammad Royan ${ }^{3}$, Tirton Nefianto ${ }^{4}$

1. Disaster Management, Indonesia Defense University, Bogor/ Indonesia

2. State Islamic University of Jakarta, South Tangerang/Indonesia

3. Mitigation and Preparedness Division of Health Crisis Center (PPKK), Surabaya/Indonesia

4. Indonesia Defense University, Sentul Bogor/Indonesia 
Study/Objective: Disasters in Indonesia have a positive trend showing that the average disaster has increased every year.

Background: isaster management circumstances can reduce the risk of disaster, but it is technically concern on pre-disaster mitigation efforts. This research was a field study in PPKK which had been done from January 12, 2015 until February 16, 2015. The purpose of the research is to know the challenges on implementation of pre-disaster efforts of PPKK in 2014.

Methods: This research was using a qualitative approach by through in-depth interviews and also from program reports of PPKK. The information and data collected then processed by Root Cause Analysis (RCA).

Results: The challenges that may caused a high incidence of health crisis in Indonesia related to the pre-disaster efforts of PPKK were that (1) inadequate amount of staff, (2) lack of control PPKK to Regional Health Department as the implementer; (3) irrelevant data analysis and display; and (4) capacity building of human resources did not involve all regionals.

\section{Conclusion: N/A}

Prehosp Disaster Med 2017;32(Suppl. 1):s67-s68

doi:10.1017/S1049023X17001820

\section{Preparing the Public Health Workforce to Meet the Challenges of Rising Sea-Levels, Virginia \\ S.M. Becker}

School Of Community \& Environmental Health, Old Dominion University - College of Health Sciences, Norfolk/United States of America

Study/Objective: The objective of this paper is to outline the key components of a new initiative in the Hampton Roads region of Virginia, aimed at preparing future public health professionals for the health challenges posed by rising sealevels.

Background: Many of the most serious impacts of rising sea levels are those affecting human health. Bigger storm surges and increased flooding can result in deaths and injuries in affected communities, population displacement and dislocation, and associated mental health impacts. Higher sea-water levels can also result in the rapid growth of mosquito populations and the spread of disease, since stagnant bodies of brackish water provide ideal breeding grounds for disease-carrying mosquitos. Examples of other health impacts include the loss of vital fresh water supplies due to the intrusion of salt-water, damage to essential healthcare and public health facilities, and the spread of biological and chemical contaminants. Because the direct and indirect health impacts of rising sea levels are expected to grow significantly in the coming years, and it is crucial that future public health professionals be trained, and have practical experience with the health issues and implications of rising sea-levels.

Methods: In the Hampton Roads region of Virginia, an area that is already experiencing significant effects from rising seas, an innovative effort has been launched to help prepare the future public health professionals to meet the health challenges of rising sea-levels. Based on the Masters of Public Health (MPH) program, sponsored jointly by Old Dominion University (ODU) and Eastern Virginia Medical School, the initiative includes several inter-related components. One part involves the incorporation into the MPH curriculum of new rising sea-level educational content and training modules. In addition, because practical experience with rising sea-level issues is also essential, classroom and related curricular efforts are being complemented with newly-developed practicum sites and other practice opportunities at agencies and organizations already grappling with real-world rising sea-level issues in the region.

Results: Initial efforts to incorporate rising sea-level content and training into the MPH curriculum began in 2014, and have continued to expand since that time. Meanwhile, close links were established with agencies and organizations currently addressing rising sea-level issues in the region, and in 2015, the first student practicum site focused specifically on rising sealevels and public health was created. Additional practicum sites and other practice opportunities related to rising sea-levels were developed in 2016 and are slated to expand further. Through this developing initiative, future public health professionals have the opportunity to learn about rising sea-levels and health issues, and be part of real-world rising sea-levels adaptive planning and preparedness activities.

Conclusion: Many of the most serious impacts of rising sea levels are those affecting human health. Thus, it is crucial that future public health professionals be familiar with, trained in, and have practical experience with, the health issues and implications of rising sea-level. The new initiative now under way in the Hampton Roads region of Virginia, though still in its early stages, is already helping prepare future public health professionals in the region to better meet the emerging health challenges posed by rising sea-levels.

Prehosp Disaster Med 2017;32(Suppl. 1):s68

doi:10.1017/S1049023X17001832

\section{Hospital Health Resources Management: Impacts and}

Legacy of a Disaster in Brazil

Ivana Trevisan ${ }^{1}$, Regina R. Witt ${ }^{2}$, Daiane Dal Pai ${ }^{2}$, Lynette Cusack ${ }^{3}$

1. Enfermagem, Hospital de Clínicas de Porto Alegre, Porto Alegre/ Brazil

2. Universidade Federal do Rio Grande do Sul, Porto Alegre/Brazil

3. The University of Adelaide, Adelaide/SA/Australia

Study/Objective: To analyze the impacts and legacy on human resource management, from the care provided to victims of a fire at a hospital in Southern Brazil.

Background: Responding to disaster situations challenges hospitals, which have to be prepared to respond to a sudden increase in emergency presentations, while still providing services to existing patients. In January 2013, a fire in a nightclub resulted in 160 people injured, 18 of which were transferred to the Hospital de Clínicas de Porto Alegre.

Methods: A qualitative case study was undertaken. This included semi-structured interviews with 17 health professionals who were involved in the clinical care of these injured victims. Also documents, such as Institutional Contingency Plans for External Catastrophes, and reports specifically related 